\title{
EXAUSTÃO EMOCIONAL EM ENFERMEIROS DE UM HOSPITAL PÚBLICO
}

\author{
Emotional exhaustion in nurses of a public hospital \\ Agotamiento emocional em enfermeros en un hospital público
}

Marina Pereira Rissardo ${ }^{1}$

Renata Cristina Gasparino ${ }^{2}$

\section{RESUMO}

0 presente estudo teve como objetivo identificar o nível de burnout nos enfermeiros de um hospital público do interior do estado de São Paulo. A pesquisa descritiva e transversal foi realizada com 69 enfermeiros de diferentes setores do hospital. Foram utilizados uma ficha para caracterização da amostra e o Inventário de Burnout de Maslach (IBM). A maioria da amostra era do sexo feminino, solteira, com pós-graduação, sem nenhum outro vínculo empregatício e lotada nas unidades de clínica médica/cirúrgica. As médias encontradas para as subescalas do intrumento foram: 21,4 para exautão emocional; 9,3 para despersonalização; e 31,3 para diminuição da realização pessoal, revelando um nível moderado da síndrome de burnout. Destaca-se a importância de se avaliar a prevalência da síndrome entre os diferentes profissionais, pois o seu desenvolvimento pode acarretar resultados negativos para os profissionais, pacientes e instituições.

Palavras-chave: Esgotamento profissional. Enfermagem. Ambiente de instituições de saúde.

\begin{abstract}
This study has the objective of identifying the level of Burnout in the nurses of a public hospital in the countryside of the Brazilian state of São Paulo. The descriptive and cross-sectional research was made with 69 nurses of different sectors of the hospital. The sample composition questionnaire and the Maslach Burnout Inventory (MBI) were used. The majority of the sample was female, single, had postgraduate degrees, did not have another formal employment contract and worked in the medical/surgical clinic. The averages that were found for the subscales of the instrument were: 21,4 for the emotional exhaustion; 9,3 for the depersonalization; and 31,3 for the personal fulfillment decrease, revealing a moderate level of the Burnout syndrome. It is underscored the importance in evaluating the prevalence of this syndrome in different professionals, because its development can cause negative results for the health care professionals, patients and institutions.
\end{abstract}

Keywords: Burnout, professiona.Nursing. Health facility environment.

\section{Resumen}

Este estudio tiene como propósito identificar el nivel del Burnout en los enfermeros de un hospital público del interior del estado brasileño de São Paulo. La investigación, descriptiva y transversal (cross-sectional), fue realizada con 69 enfermeros de diferentes sectores del hospital. Fueron utilizadas cédulas de caracterización de la amuestra y el Inventario de Burnout de Maslach (IBM). La mayoría de la amuestra era de mujeres, solteras, con posgrado, no poseían otro vínculo laboral y trabajaban en la unidad de clínica médica/quirúrgica. Las medias encontradas para las subescalas del instrumento fueron: 21,4 para el agotamiento emocional; 9,3 para despersonalización; y 31,3 para la disminución de la realización personal, revelando un nivel moderado del Síndrome Burnout. Se destaca la importancia de evaluar la prevalencia del síndrome entre los diferentes profesionales, porque su desenvolvimiento puede causar resultados negativos para los profesionales, pacientes e instituciones.

Palabras clave: Agotamiento profesional. Enfermería. Ambiente de instituciones de salud. 


\section{INTRODUÇÃO}

As mudanças tecnológicas possibilitaram às empresas 0 aumento da produtividade e, consequentemente, dos lucros; porém, esse desenvolvimento acarretou impactos negativos à saúde do trabalhador, com manifestações tanto na esfera física quanto psíquica. 0 surgimento de novas enfermidades relacionadas às mudanças introduzidas no mundo do trabalho tem sido muito apontado nas produções científicas das últimas décadas".

0 estresse no trabalho é decorrente da inserção do indivíduo nesse contexto, pois o trabalho pode representar fonte de satisfação ou insatisfação pessoal2. Isso ocorre quando o ambiente de trabalho é percebido como uma ameaça ao indivíduo, repercutindo no plano pessoal e profissional, com demandas maiores do que a sua capacidade de enfrentamento ${ }^{3}$.

A Enfermagem foi classificada, pela Health Education Authority ${ }^{A}$, como a quarta profissão mais estressante no setor público, devido ao constante contato com doenças, o que expõe a equipe a fatores de risco de natureza física, química, biológica e psíquica. A complexidade dos inúmeros procedimentos realizados pela equipe, o grau de responsabilidade nas tomadas de decisão, a falta de recursos humanos, os possíveis acidentes de trabalho e o trabalho por turnos aumentam a angústia e a ansiedade dos profissionais, desencadeando, muitas vezes, situações de estresse ${ }^{5}$.

A baixa remuneração, associada ao excesso de volume de trabalho, a diversidade de tarefas e 0 apoio insuficiente geram conflitos e constituem-se em importantes desencadeadores da síndrome de burnout nos profissionais enfermeiros ${ }^{1}$.

Por ser altamente estressante, o trabalho de enfermagem pode ter como consequência a desmotivação, insatisfação, aumento da taxa de absenteísmo e, até mesmo, 0 abandono da profissão $0^{5,6}$. Diante desta problemática, torna-se necessário que as instituições tenham um planejamento, a fim de combater o burnout, fazendo com que os profissionais se sintam valorizados, motivados e, principalmente, trabalhem em um ambiente harmonioso e com recursos técnicos e humanos que favoreçam o desenvolvimento de suas atribuições.

Quando o estresse vivenciado pelo profissional em seu ambiente de trabalho se torna crônico, passa a ser chamado de burnout, ou síndrome de Burnout, que vem do inglês e é utilizado para designar algo ou alguém que não possui mais energia? .

A primeira utilização do termo burnoutse deu em 1974 por Freudenberger, um psicólogo, que o descreveu como sendo um sentimento de fracasso e exaustão causados por um excessivo desgaste de energia e de recursos ${ }^{2}$, observado por meio do sofrimento existente entre os profissionais que trabalhavam diretamente com pacientes dependentes de substâncias químicas'.

0 burnouté uma reação cumulativa aos estressores ocupacionais contínuos, sendo caracterizado por cronicidade, ruptura da adaptação, desenvolvimento de atitudes negativas e também comportamentos de redução da realização pessoal no trabalho ${ }^{8}$.

Na síndrome de burnout estão envolvidas atitudes e condutas negativas com relação aos usuários, clientes, organização e trabalho. É um processo gradual, de uma experiência subjetiva, envolve atitudes e sentimentos que acarretam problemas de ordem prática e emocional ao trabalhador e à organização. Ocorre quando o lado humano do trabalho não é considerado; já no estresse, não estão envolvidas tais atitudes e condutas, pois se trata de um esgotamento pessoal com interferência apenas na vida do indivíduo, e não necessariamente na sua relação com o trabalho'.

Portanto, burnouté uma síndrome em que o profissional perde o sentido da sua relação com o trabalho e sente como se as coisas já não tivessem mais importância. Trata-se de um conceito que envolve três dimensões, que podem aparecer associadas, mas que são independentes: exaustão emocional; despersonalização; e falta de envolvimento no trabalho ou diminuição da realização pessoal ${ }^{1,9}$.

A exaustão emocional é definida por uma diminuição ou ausência de energia associada a um sentimento de esgotamento emocional. A manifestação pode ser física, psíquica ou uma combinação delas. Os trabalhadores percebem que já não têm condições de empregar mais energia para prestar uma assistência para seu cliente e familiares ${ }^{1}$.

A despersonalização pode ser conceituada como uma insensibilidade emocional em que prevalece a dissimulação afetiva. Nessa dimensão, são manifestações comuns a ansiedade, o aumento da irritabilidade, a desmotivação, a redução dos objetivos e do comprometimento com os resultados do trabalho, além da redução do idealismo, alienação e egoísmo'.

A falta de envolvimento pessoal no trabalho ou diminuição da realização pessoal é uma dimensão em que existe um sentimento de inadequação pessoal e profissional. Há uma tendência de se realizar uma autoavaliação negativa, o que pode afetar a realização do trabalho e 0 atendimento dos pacientes, bem como o comprometimento com a instituição'.

Os primeiros sentimentos negativos são direcionados aos desencadeantes do processo, ou seja, clientes e colegas de trabalho; posteriormente atingem amigos e familiares e, por último, o próprio profissional. Como sintomas físicos e possíveis consequências dessa síndrome, podem ser observados: cefaleia, alterações gastrointestinais, absenteísmo, intenção de sair do emprego, baixa produtividade, aumento da rotatividade, diminuição da satisfação com o trabalho ${ }^{10}$, e, como sintomas psíquicos: ansiedade, depressão, desmotivação, frustração, medo, raiva, hostilidade, diminuição da autoestima e distúrbios do sono ${ }^{11}$.

$\mathrm{Na}$ Classificação Internacional de Doenças, o Esgotamento é reconhecido pelo código Z73.0, e, no Brasil, há um regulamento da previdência social que reconhece a Sensação de Estar Acabado como sinônimo da Síndrome de Burnoute 
como um transtorno mental relacionado com o trabalho $0^{12}$. Por isso, essa síndrome pode acometer indivíduos de qualquer realidade social, educacional ou cultural. É preciso compreender que as mudanças no ambiente de trabalho afetam o bem-estar físico e mental dos trabalhadores e dos grupos sociais dos quais eles fazem parte'.

Diante do exposto, o objetivo do presente estudo foi identificar o nível de burnout dos enfermeiros de um hospital público do interior do Estado de São Paulo.

\section{MÉTODO}

0 estudo, de caráter descritivo e transversal, foi realizado nas unidades de internação de um hospital público e filantrópico do interior do estado de São Paulo, com 218 leitos e uma média de 24 mil atendimentos por mês, sendo $99 \%$ destes atendimentos realizados pelo Sistema Único de Saúde.

A amostra foi composta por todos aqueles que atenderam aos seguintes critérios de inclusão: ser enfermeiro, prestar assistência direta aos pacientes; não estar de férias ou licença; aceitar participar da pesquisa e assinar o Termo de Consentimento Livre e Esclarecido (TCLE).

Para a coleta de dados, os enfermeiros receberam uma ficha para caracterização da amostra e o Inventário de "Burnout" de Maslach (IBM). A ficha para caracterização da amostra foi elaborada pelas pesquisadoras e abordou características pessoais e profissionais dos enfermeiros.

0 Inventário de Burnout de Maslach foi adaptado e validado por Tamayo ${ }^{13}$, para a cultura brasileira, e tem por objetivo mensurar o desgaste físico e mental do trabalhador, por meio da avaliação da frequência com que o profissional vivencia determinadas situações em relação ao seu trabalho. É um instrumento autoaplicável, composto por 22 itens agrupados em três dimensões: exaustão emocional (nove itens), diminuição da realização pessoal (oito itens) e despersonalização (cinco itens) ${ }^{13}$.

Os itens são avaliados por uma escala tipo Likert com cinco pontos: um (nunca); dois (raramente); três (algumas vezes); quatro (frequentemente) e cinco (sempre). Para avaliar as dimensões exaustão emocional, despersonalização e diminuição da realização pessoal, obtém-se a soma das respostas dos sujeitos para cada subescala, a qual pode variar entre 9 e 45 pontos para a subescala exaustão, entre 5 e 25 pontos para a subescala despersonalização e entre 8 e 40 pontos para a subescala diminuição da realização pessoal. Nas duas primeiras subescalas, quanto maior for a pontuação, maior será o sentimento vivenciado pelo profissional; já na subescala diminuição da realização pessoal, quanto maior a pontuação, menor será o sentimento vivenciado pelo profissional enfermeiro, pois o escore é inverso ${ }^{13}$.

Com relação às subescalas do instrumento, um nível moderado de burnouté representado em escores médios nas três subescalas. Um baixo nível de burnouté representado por baixos escores nas subescalas exaustão emocional e despersonalização e altos escores na subescala diminuição da realização pessoal e um alto nível de burnouté representado por altos escores nas subescalas exaustão e despersonalização e baixos escores na subescala diminuição da realização pessoal ${ }^{13}$.

Os nomes dos enfermeiros foram obtidos com a diretoria do hospital, e a coleta de dados foi realizada nos meses de maio e junho de 2011. Os sujeitos foram abordados de forma individual, em suas unidades de trabalho, onde foram explicados os objetivos da pesquisa, e aqueles que concordaram em participar assinaram o TCLE. Os participantes, após a assinatura, receberam individualmente um envelope contendo a ficha de caracterização e o IBM. Os envelopes foram entregues à pesquisadora imediatamente após seu preenchimento ou em uma data combinada.

0 projeto foi aprovado pelo Comitê de Ética em Pesquisa da Faculdade de Medicina de Jundiaí (FMJ) (Protocolo n ${ }^{0} 53 /$ 11) e pela diretoria da instituição onde foi realizada a pesquisa. De acordo com os procedimentos éticos envolvidos em pesquisas com seres humanos, foram esclarecidos a não obrigatoriedade da participação, os objetivos do trabalho e a garantia de anonimato.

Os dados foram analisados utilizando-se o programa Statistical Analysis Software (SAS) versão 9.2. As variáveis categóricas foram avaliadas por meio de análise descritiva e as contínuas, por meio de medidas de posição.

Para classificar os níveis de burnout em alto, moderado e baixo, foram determinados os percentis 33 e 67 da curva, para cada subescala. A associação entre as subescalas e as variáveis em estudo foi avaliada por meio dos testes de Quiquadrado e exato de Fisher.

A confiabilidade do total de itens foi verificada por meio da consistência interna com o cálculo do coeficiente alfa de Cronbach, no qual se considerou como limite inferior aceitável o valor de $0,70.0$ nível de significância assumido foi de $5 \%$, isto é, $p$-valor $<0,05$.

\section{RESULTADOS}

A amostra foi composta por 69 participantes que corresponde a $94,5 \%$ do total de enfermeiros da instituição, com uma média de 32 anos de idade (DP $\pm 5,98$ ). A maioria era do sexo feminino $(73,9 \%)$, solteira $(55,1 \%)$, com especialização em Enfermagem (72,1\%), não tinha outro vínculo empregatício (72,5\%), já havia tirado férias no último ano $(88,4 \%)$ e tinha algum tipo de hobby $(70,6 \%)$.

Grande parte dos sujeitos trabalhavam como enfermeiros há menos de 10 anos (82,6\%) e, na instituição da pesquisa, há menos de cinco anos (38,2\%). No que se refere ao setor, a maior parte estava lotada nas unidades de Clínicas Médica/Cirúrgica (40,4\%), seguida das Unidades de Terapia Intensiva (28,9\%) e das unidades de Urgência e Emergência 
(21,7\%). Com relação ao turno de trabalho, 43,5\% realizavam plantão noturno e tinham sob sua responsabilidade diária uma média de 33 pacientes (DP \pm 47).
Com relação às subescalas do instrumento, as médias e o desvio-padrão estão apresentadas na Tabela 1.

Tabela 1. Média e desvio-padrão das subescalas do Inventário de Burnout de Maslach. Jundiaí, 2011

\begin{tabular}{lcc} 
Tabela 1. Média e desvio-padrão das subescalas do lnventário de Burnout de Maslach. Jundiaí, 2011 & Média & DP* \\
\hline Subescalas & 21,4 & $\pm 5,5$ \\
\hline Exaustão emocional & 9,3 & $\pm 2,6$ \\
Despersonalização & 31,3 & $\pm 3,7$ \\
\hline Diminuição da realização pessoal (escore inverso) &
\end{tabular}

*DP: Desvio-padrão.

Ao analisar as médias das subescalas, constatouse que a amostra apresenta moderado nível de exaustão emocional, despersonalização e diminuição da realização pessoal, representando, assim, um nível moderado da síndrome de burnout.

Associando a subescala exaustão emocional com o turno de trabalho, observou-se que os enfermeiros que trabalham à noite se sentem mais exaustos emocionalmente do que os que trabalham durante o dia $(p=0,0171)$.

Nas escalas despersonalização e diminuição da realização pessoal não foram encontradas associações significativas com relação às características sociodemográficas e profissionais.

No que se refere à consistência interna, o instrumento obteve um coeficiente de alfa de Cronbach de 0,81.

\section{DISCUSSÃO}

No presente estudo, a predominância do sexo feminino pode ser explicada pela própria composição desta equipe profissional, a qual vem historicamente associada à atividade religiosa (feminização do cuidado), a aspectos culturais, trazendo em sua trajetória o cuidado, que sempre foi uma função das mulheres. Nos últimos anos, tem-se observado um aumento no número de homens, mas, ainda assim, a Enfermagem é considerada, pela sociedade, uma profissão predominantemente feminina ${ }^{14}$.

Quando questionados sobre a realização de cursos de pós-graduação, foram obtidos resultados semelhantes aos de outros estudos ${ }^{9,15}$, em que a maioria dos participantes teve alguma especialização em sua área de atuação. Nos últimos anos, houve um importante crescimento na quantidade de cursos de graduação em Enfermagem no Brasil, aumentando a disponibilidade de profissionais, o que ocasionou a busca por aprimoramento a fim de se conseguir a inserção no mercado de trabalho ${ }^{16}$.

A maioria dos participantes era jovem e por isso solteira e com pouco tempo de experiência na profissãa $0^{9,13}$. Além disso, principalmente as mulheres com um grau de escolarização mais elevado aguardam por uma estabilização econômica e profissional para realizarem o matrimônio.
Verifica-se que a maioria dos pesquisados, assim como em outros estudos ${ }^{9,15}$, pertenciam às unidades de clínicas médica ou cirúrgica, pois essas áreas eram as maiores do hospital, necessitando, portanto, de mais profissionais para desenvolver a assistência.

No presente estudo, os enfermeiros tinham uma média diária alta de pacientes sob seus cuidados. Isso não foi observado em outra pesquisa nacional15. Esta relação de pacientes por profissional pode ser explicada em razão de um número considerável de participantes atuar nas unidades de urgência e emergência, onde a rotatividade de pacientes é bastante alta e não há um número fixo de leitos como acontece nas unidades de internação.

A remuneração dos profissionais enfermeiros é baixa, 0 que os obriga a ter mais de um vínculo empregatício, ocasionando uma sobrecarga de trabalho que pode comprometer a saúde física e psíquica ${ }^{6}$; porém, a maior parte da amostra não tinha outro vínculo empregatício, apenas seu trabalho nesta instituição como fonte de renda, como também foi mostrado em outras pesquisas ${ }^{15,17}$.

Os profissionais do presente estudo encontravam-se com um nível moderado de burnout, assim como constatado em outra pesquisa ${ }^{9}$. Observaram-se maiores níveis de exaustão emocional nos enfermeiros do plantão noturno, talvez pela alteração no ritmo biológico e pelo sono perdido não poder ser adequadamente recompensado, o que consequentemente leva a uma diminuição da capacidade mental e física ${ }^{18}$.

\section{CONCLUSÃO}

0 nível de burnout encontrado nos enfermeiros do presente estudo foi moderado. 0 avanço da tecnologia implica mudanças no ambiente de trabalho que afetam o bem-estar físico e mental dos trabalhadores, e, por isso, a síndrome de burnoutprecisa ser considerada um problema de saúde pública.

Destaca-se a importância de se avaliar a síndrome entre as diversas categorias de profissionais de enfermagem a fim de que as instituições possam adotar estratégias que contribuam para melhorias no ambiente de trabalho, tornando-o menos estressante, pois os custos do desenvolvimento e manifestação do burnout são significativos não somente para os profissionais, mas também para os pacientes, instituições e sociedade. 


\section{REFERÊNCIAS}

1 Murofuse NT, Abranches SS, Napoleão AA. Reflexões sobre estresse e burnout e a relação com a enfermagem. Rev. Latino-Am. Enfermagem. $2005 \mathrm{mar} / \mathrm{abr} ;$ 13(2): 255-61.

2Silva DCM, Loureiro MF, Peres RS. Burnoutem profissionais de enfermagem no contexto hospitalar. Psicol. hosp. 2008 abr; 6(1): 39-51.

3Meneguini F, Paz AA, Lautert L. Fatores ocupacionais associados aos componentes da síndrome de burnoutem trabalhadores de enfermagem. Texto \& contexto enferm. 2011 abr/jun; 20(2): 225-33.

40rtiz GCM, Platiño NAM. El stress y su relación com las condiciones de trabajo del personal de enfermaría. Invest. educ. enferm. 1991; 9(2): 8399.

5Murofuse NT, Abranches S, Napoleão AA. Reflexões sobre estresse e burnout e a relação com a enfermagem. Latino-Am. Enfermagem. 2005 mar/abr; 13(2): 255-61.

6Stacciarini JMR, Tróccoli BT. Occupational stress and constructive thinking: health and job satisfaction. J. adv. nurs.. 2004; 46(5): 480-7.

7Grazziano ES, Ferraz Bianchi ER. Impacto del estrés ocupacional y burnout em enfermeros. Enferm. glob. 2010; (18): 2-5.

8Lima FD, Buunk AP, Araújo MBJ, Chaves JGM, Muniz DLO, Queiroz LB. Síndrome de burnout em residentes da Universidade Federal de Uberlândia. 2004. Rev. bras. educ. méd. 2007 maio/ago; 31(2): 137-46.

9Lautert L. 0 desgaste profissional do enfermeiro [tese de mestrado]. Salamenca (ESP): Universidade Pontifícia de Salamanca, 1995.

10 McVicar A. Workplace stress in nursing: a literature review. J. adv. nurs.. 2003; 44(6): 633-42.

11 Tucunduva, et al. A síndrome da estafa profissional em médicos cancerologistas brasileiros. Rev. Assoc. Med. Bras. 2006; 52(2): 108-12.

12 Trigo TR, Teng CT, Hallak JEC. Síndrome de burnout ou estafa profissional e os transtornos psiquiátricos. Rev. psiquiatr. clín. 2007; 34(5): 223-33.

13 Tamayo MR. Relação entre a síndrome do burnout e os valores organizacionais no pessoal de enfermagem de dois hospitais públicos [dissertação de mestrado]. Brasilia (DF): Universidade Federal de Braślia; 1997.

14 Martino MMF, Misko MD. Estados emocionais de enfermeiros no desempenho profissional em unidades críticas. Rev. Esc. Enferm. USP. 2004 jun; 38(2): 161-7.

15Gasparino RC. Adaptação cultural e validação do instrumento "nursing work index - revised” para a cultura brasileira [dissertação de mestrado]. Campinas (SP): Universidade Estadual de Campinas; 2008.
16Silva EM, Baragatti DY, Figueira MCS, Lima RS, Silva TC. 0 aumento dos cursos de graduação em enfermagem no Brasil nos últimos 15 anos: determinações e sustentabilidade. $61^{\circ}$ Congresso Brasileiro de Enfermagem; 2009 dez 7-10; Fortaleza (CE), Brasil. ABEn; 2009. p. 2345-8. (Anais)

17Meneghini F, Paz AA, Lautert L. Fatores ocupacionais associados aos componentes da síndrome de burnoutem trabalhadores de enfermagem. Texto \& contexto enferm. $2011 \mathrm{abr} / \mathrm{jun} ;$ 20(2): 225-33.

18Silva RM, BeckCLC, Magnago TSBS, Carmagnani MIS, Tavares JP, Prestes FC. Trabalho noturno e a repercussão na saúde dos enfermeiros. Esc Anna Nery. 2011 abr/jun; 15(2): 270-6. 